\section{SEXUAL BEHAVIOUR IN YOUNGER MSM AND SELF- PERCEIVED SEXUAL RISK USING A LOCAL HIV RISK ASSESSMENT TOOL (HIVRAT)}

doi:10.1136/sextrans-2012-050601a.17

\section{J Alagaratnam, ${ }^{*}$ A Black, A Smith. St Mary's Hospital}

Background HIV infection continues to disproportionately affect MSM in the UK. The 2011 HPA report "Sexually Transmitted Infections in MSM in the UK" highlights the need for one-to-one behavioural interventions. Thus, identifying those at highest risk is essential.

Aims To profile the sexual behaviour of younger MSM attending a dedicated clinic. To establish how MSM perceive their sexual risk and explore the use of a simple HIV Risk Assessment Tool (HIVRAT).

Methods MSM attending a weekly clinic offering HIV testing selfcomplete a 6 question HIVRAT in addition to standard history. The HIVRAT records number of male partners in previous 12 months, and number of unprotected anal intercourse (UPAI) partners (previous 3 and 12 months). It also contains a Likert scale of perceived sexual risk. Data was collected over 6 months from June 2011. Statistical analysis was performed in Excel and correlated using Spearman's Rank methodology.

Results 138 men completed the HIVRAT (aged 18-35). Median number of sexual partners in preceding 12 months $=8$ (range $1-250,42 \%$ reported $>10$ partners). Median number of UPAI partners in preceding 3 months $=0$ (range $0-5$ ) and 12 months $=1$ (range $0-8)$. Perceived risk was scored as $1=$ Very low (20.4\%), 2 (44.5\%), 3 (28.5\%), $4(4.4 \%)$ and $5=$ Very high (2.2\%). There was poor correlation between sexual behaviour and perceived risk. For MSM who had UPAI with one or more partners in the previous 3 and 12 months, there was a moderately positive correlation between actual risk and perceived risk (SRCC 0.517 and 0.544 respectively).

Conclusions Only $6.6 \%$ of MSM judged their personal HIV risk as high in a cohort where $36 \%$ reported UPAI with two or more partners in 12 months. Tools like HIVRAT provide valuable information which is not routinely collected. Asking about UPAI during the 12-month period prior to testing showed the strongest correlation between actual and perceived risk, and could help identify MSM who would benefit most from behavioural intervention.

\section{SEXUAL BEHAVIOUR, PARTNERSHIP PATTERNS AND STI DIAGNOSES AMONG HIV POSITIVE MSM: IMPLICATIONS FOR HIV/STIS TRANSMISSION AND PARTNER NOTIFICATION}

doi:10.1136/sextrans-2012-050601a.18

${ }^{1} \mathrm{~S}$ Wayal, 'G Hart, ${ }^{1} \mathrm{~A}$ Copas, ${ }^{2} \mathrm{~S}$ Edwards, ${ }^{3} \mathrm{JA}$ Cassell.. ${ }^{1}$ University College London, London, UK; ${ }^{2}$ NHS Camden Provider Services, London, UK; ${ }^{3}$ Brighton and Sussex Medical School, Brighton, UK

Aims In the UK, HIV+ve men who have sex with men (MSM) are disproportionately affected with sexually transmitted infections (STIs). STIs can enhance HIV transmission. We examined factors associated with STIs diagnoses and partner notification, and explored preferred methods for STIs notification among HIV+ve MSM.

Methods 429 HIV+ve MSM attending a central London HIV clinic completed a computer-assisted survey (May-September 2010). Multivariate logistic regression analyses were conducted.

Results $86 \%$ men (368/429) were sexually active in the last year. Of these sexually active men, 84\% (305/362) had tested for STIs.
Among men who tested for STIs, 57\% (174/305) reported engaging in unprotected anal intercourse (UAI) and 32\% (98/305) were diagnosed with STIs. UAI, particularly non-concordant unprotected anal intercourse, age $<35$ years, concurrent sexual partnerships were independently associated with STIs diagnoses. $58 \%$ men had notified ¡Ý1 partner following STIs diagnosis. Being employed, born in the UK, concerns about breach of HIV confidentiality were negatively associated; while clinic advice and support were positively associated with partner notification following STIs diagnosis. 79\% (339/429) men reported willingness to notify partners of STIs in the future. Of these, $76 \%$ men were willing to notify a boyfriend themselves. $11 \%$ men expressed preference for provider referral. Most men were willing to notify regular partners by phone. Men expressed willingness to notify casual partners by phone, text message, or anonymous provider-led methods.

Conclusions The high level of risky sexual behaviour, STIs diagnosis and its association with non-concordant unprotected anal intercourse among HIV+ve MSM suggests that partner notification provides opportunities for HIV and STIs case-finding and treatment. The offer of a choice of notification methods to HIV+ve MSM, particularly young men and men with multiple/concurrent, casual partners, may override personal, partnership, and structural barriers to partner notification.

\section{SEXUAL PARTNERSHIP PATTERNS AND SEXUALLY TRANSMITTED INFECTIONS IN HIV POSITIVE MEN WHO HAVE SEX WITH MEN: IMPLICATIONS FOR PARTNER NOTIFICATION}

doi:10.1136/sextrans-2012-050601a.19

${ }^{1} \mathrm{~S}$ Wayal, * ${ }^{2} \mathrm{~J}$ A Cassell, ${ }^{3} \mathrm{~S}$ Edwards, ${ }^{1} \mathrm{G}$ Hart. ${ }^{1}$ University College London, London, UK; ${ }^{2}$ Brighton and Sussex Medical School, Brighton, UK; ${ }^{3}$ NHS Camden Provider Services, London, UK

Aims It is essential to understand the implications of sexual partnerships, sexual networks and type of sexually transmitted infections (STIs) on partner notification to maximise its effectiveness. We examined the relationship of these factors with HIV+ve men who have sex with men's (MSM) attitudes towards partner notification.

Methods 24 purposively selected men participated in semistructured interviews (May 2010-February 2011). Piloted vignettes about different types of STIs diagnosis and sexual partners were used to facilitate discussion. Framework analysis was conducted.

Results Men perceived the personal and public health benefits of HIV/STIs notification. However, HIV was perceived as a "fatal illness" and partner notification as "imperative" to facilitate partners' access to antiretroviral treatment. Some men preferred immediate HIV partner notification to allow post-exposure prophylaxis; others preferred to wait due to their own emotional burden of HIV diagnosis. Men perceived HIV notification with greater fear of stigma and "blame culture" than STIs notification. Concerns regarding breach of confidentiality and long-term relationships were perceived barriers to HIV partner notification, while clinic advice and support was favoured. Men who intentionally engaged in bareback sex perceived STIs partner notification as "the norm". However, some men emphasised their and partners' "personal responsibility" to test for STIs regularly. Men favoured notifying a boyfriend and regular partners personally, especially for non-curable STIs like Hepatitis C (HCV). However, fear of being blamed or blaming group/casual sex partners, especially for HCV, were barriers to STIs notification. Provider-led or anonymous-notification methods were preferred in such cases.

Conclusions HIV+ve MSM should be offered clinic support for patient-led HIV notification, provider-led methods for HCV 
notification, and notifying group/casual sex partners to improve partner notification.

\section{HOME SAMPLING THROUGH SOCIAL NETWORK WEBSITES: CAN WE REDUCE UNDIAGNOSED HIV?}

doi:10.1136/sextrans-2012-050601a.20

${ }^{1} \mathrm{E}$ Elliot, ${ }^{*} \mathrm{~A}$ McCowan, ${ }^{1} \mathrm{~S}$ McCormak, ${ }^{1} \mathrm{M}$ Rossi, ${ }^{2} \mathrm{~T}$ Van Every. ${ }^{1}$ Chelsea and Westminster Hospital, London, UK; ${ }^{2} \operatorname{Dr}$ Thom

Background Approximately 1 in 20 men who have sex with men (MSM) in London has undiagnosed HIV. It is a national priority to minimise time spent undiagnosed to reduce morbidity, mortality and onward transmission. Sigma reported that $29 \%$ of MSM recruited online in 2008 had never tested. We piloted an offer of home HIV sampling for MSM in partnership with the Gaydar social network website.

Objectives The objectives of the pilot were to determine (1) uptake of an online HIV risk self-assessment (2) uptake of offer for home testing (3) the proportion of those completing the assessment that is high risk.

Methods Each day 200 MSM in London were invited by Gaydar to assess their HIV risk. "High risk" was defined as unprotected anal sex (UAI) with HIV + partner or of unknown HIV status. Following assessment but regardless of risk, users were offered a postal HIV salivary sampling kit. Reactive results were confirmed in the clinic. Data were analysed using Google Analytics.

Results 4500 invites were issued between 7 November 2011 and 11 January 2012. 363 (8\%) clicked through to the project website, 321 (88\%) of whom completed the HIV risk assessment. Of these, 146 (45\%) were defined as "high risk". 209 clicked through for information on home sampling, 82 (39\%) of whom had never HIV tested. 132 test kits were requested, 73 (55\%) were returned, 4 of which were new HIV diagnoses ( $3 \%$ of kits sent and $5.5 \%$ of samples returned).

Discussion Although low, the uptake appears to reach a population "less tested" than the population that took part in the Sigma online survey or typical of GUM clinics. The level of risk identified was similar to a clinic population with almost half reporting UAI. $55 \%$ of kits were returned and we report $5.5 \%$ or $1: 18$ new diagnoses. The project is now scaling up to message all 120000 Gaydar users in Central London over 12 months and aims to provide evidence to inform the potential roll out of online strategies to enhance community based HIV testing in the UK.
SHOULD WE OPPORTUNISTICALLY TREAT RECTAL CHLAMYDIA TRACHOMATIS INFECTION IN MEN WHO HAVE SEX WITH MEN PRESENTING WITH NON-SPECIFIC URETHRITIS?

doi:10.1136/sextrans-2012-050601a.21

${ }^{1} \mathrm{M}$ Rayment, ${ }^{1} \mathrm{~L}$ Bull, ${ }^{*}{ }^{2} \mathrm{~S}$ Mandalia, ${ }^{1} \mathrm{~F}$ Boag, ${ }^{1} \mathrm{~N}$ Nwokolo, ${ }^{1} \mathrm{R}$ Jones. ${ }^{1}$ Chelsea and Westminster Hospital NHS Foundation Trust, London, UK; ${ }^{2}$ Imperial College

Background Infection of the rectum with Chlamydia trachomatis (CT) is highly prevalent among men who have sex with men (MSM) attending our service (c. 8\%). Our guidelines advise different antibiotics for the treatment of non-specific urethritis (NSU) (azithromycin $1 \mathrm{~g}$ as a single dose) and for rectal CT infection (doxycycline $100 \mathrm{mg}$ twice daily for 7 days). MSM receiving treatment for NSU require recall if they are latterly found to have concurrent rectal CT. Should we therefore use doxycycline first line in MSM presenting with NSU?

Aims To determine the proportion of MSM diagnosed with NSU with rectal CT infection; to identify independent predictors of having rectal CT.

Methods The clinic database identified sequential MSM diagnosed with NSU over 6 months. Co-variables collected: age, sexual history, HIV status, contact history and the presence of rectal symptoms. Only men accepting rectal sampling were included. Univariate and multivariable logistic regression analyses were undertaken.

Results 566 MSM presenting with NSU were identified, of whom $130(23 \%)$ were diagnosed with CT infection of at least one anatomical site. Of this group, 99 were included in the analysis. The overall prevalence of rectal CT infection was 38\% (38/99). In men reporting a history of unprotected receptive anal intercourse (UPRAI) in the preceding 6 months, the prevalence was 61\% (31/ 51); in those without it was 7.1\% (14/48). In MSM reporting rectal symptoms, the prevalence was $90 \%(9 / 10)$; in those without: $33 \%$ (29/89). In the multivariable model, the only independent predictors of risk of rectal CT infection were UPRAI and rectal symptoms (see Abstract O21 table 1).

Discussion First line treatment of NSU in MSM could be modified based on the likelihood of concurrent CT infection. In men reporting UPRAI in the preceding 6 months, or reporting rectal symptoms, it would be reasonable to offer treatment of NSU with doxycycline. In all other patients, we propose continuing to use azithromycin first line, with subsequent recall for further treatment as necessary.

Abstract 021 Table 1 Rate of rectal chlamydia according to history and rectal symptoms

\begin{tabular}{lllllll}
\hline Variable & & Total $(\mathbf{n = 9 9 )}$ & $\begin{array}{l}\text { Number with rectal } \\
\mathbf{C T}(\%)(\mathbf{n = 3 8})\end{array}$ & Adjusted RR & $\mathbf{9 5 \%} \mathbf{~ C l}$ & $\begin{array}{l}\text { Wald statistics, } \\
\mathbf{p ~ V a l u e ~}\end{array}$ \\
\hline $\begin{array}{l}\text { History of unprotected receptive } \\
\text { anal intercourse (UPRAl) }\end{array}$ & $\mathrm{Y}$ & 51 & $31(60.8)$ & 1 & & 0.002 \\
& $\mathrm{~N}$ & 48 & $7(14.6)$ & 0.25 & $(0.07$ to 0.82$)$ \\
Rectal symptoms & $\mathrm{Y}$ & 10 & $9(90)$ & 9.54 & $(1.09$ to 83.4$)$ & 0.041 \\
& $\mathrm{~N}$ & 89 & $29(32.6)$ & 1 & & \\
\hline
\end{tabular}

